\title{
Final Focus Spot Size in a Solenoid Focusing System
}

Chen, Yu-Jiuan

\section{April 8, 2003}

Law rence

Livermore

National

Laboratory

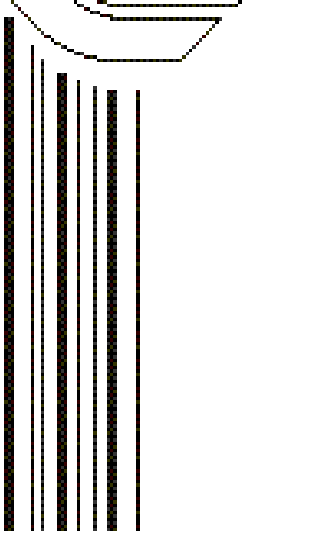




\section{DISCLAIMER}

This document was prepared as an account of work sponsored by an agency of the United States Government. Neither the United States Government nor the University of California nor any of their employees, makes any warranty, express or implied, or assumes any legal liability or responsibility for the accuracy, completeness, or usefulness of any information, apparatus, product, or process disclosed, or represents that its use would not infringe privately owned rights. Reference herein to any specific commercial product, process, or service by trade name, trademark, manufacturer, or otherwise, does not necessarily constitute or imply its endorsement, recommendation, or favoring by the United States Government or the University of California. The views and opinions of authors expressed herein do not necessarily state or reflect those of the United States Government or the University of California, and shall not be used for advertising or product endorsement purposes.

Work performed under the auspices of the U. S. Department of Energy by the University of California Lawrence Livermore National Laboratory under Contract W-7405-Eng-48.

This report has been reproduced directly from the best available copy.

Available to DOE and DOE contractors from the

Office of Scientific and Technical Information

P.O. Box 62, Oak Ridge, TN 37831

Prices available from (423) 576-8401

http://apollo.osti.gov/bridge/

Available to the public from the National Technical Information Service

U.S. Department of Commerce 5285 Port Royal Rd., Springfield, VA 22161

http://www.ntis.gov/

OR

Lawrence Livermore National Laboratory Technical Information Department's Digital Library http://www.llnl.gov/tid/Library.html 


\section{USPAS P671C Induction Accelerators}

\section{Final Focus Spot Size in a Solenoid Focusing System ${ }^{1}$}

\section{Yu-Jiuan Chen}

A linear lens can focus a cold beam to a singular point. Unfortunately, this ideal situation would never occur in the real world. Besides nonlinearity of the lens, any deviation of the beam parameters from the ideal beam's nominal beam parameters would lead to nonzero final spot size. In other words, the final spot size of a beam focused by a focusing lens with a given focusing strength depends on its beam parameters, such as the emittance, variations in beam current, energy, envelope and envelope slopes, and nonlinearity of the focusing lens. There are many types of final focusing systems. We consider only the system using a "thin" solenoid lens in this notes. Generally, the net focusing force in a solenoid focusing system is not sensitive to the beam current for an emittance dominated beam. For simplicity, we will ignore the space charge forces in the discussion, and focus on the contributions of beam emittance, energy variation and nonlinearity of the lens to the final spot size here.

\section{Spot Size and Emittance}

The final spot size $R_{f}$ caused by beam emittance can be determined by solving the RMS envelope equation. The region between the focusing lens and the focal point is a drift space. The Lee-Cooper's envelope equation for an emittance dominated, coasting beam is

$$
R^{\prime \prime}=\frac{E^{2}}{R^{3}}
$$

where $E$ is the unnormalized RMS emittance. Integrating the equation after multiplying both sides by R' yields

$$
R^{\prime 2}-R_{o}^{\prime 2}=\frac{E^{2}}{R_{o}^{2}}-\frac{E^{2}}{R^{2}},
$$

where the subscript "o" represents the beam at the exit of the solenoid lens. For a thin lens system, $R_{o}$ is also the beam radius entering the final focusing lens. At the focal point f, $R_{f}{ }^{\prime}=0$. We can rewrite Eq. (I.2) at the focal point as

$$
R_{f}^{2}=\frac{E^{2} R_{o}^{2}}{R_{o}^{2} R_{o}^{\prime 2}+E^{2}} \approx\left(\frac{E}{\left|R_{o}^{\prime}\right|}\right)^{2} .
$$

\footnotetext{
${ }^{1}$ This work was performed under the auspices of the U.S. Department of Energy by University of California Lawrence Livermore National Laboratory under contract No. W-7405-Eng-48.
} 
To get the final result above, we have assumed $R_{o}\left|R_{o}{ }^{\prime}\right| \gg E$ since the final spot size is usually much less than the beam size entering the final focusing lens. Equation (I.3) shows that the final spot size would be zero if the beam were cold. From the beam envelope of a cold beam shown in Fig. 1, it is obvious that $R_{o}{ }^{\prime}=-R_{d} / f$. Therefore, the final spot size caused by emittance is given by $R_{f} \cong E f / R_{o}$.

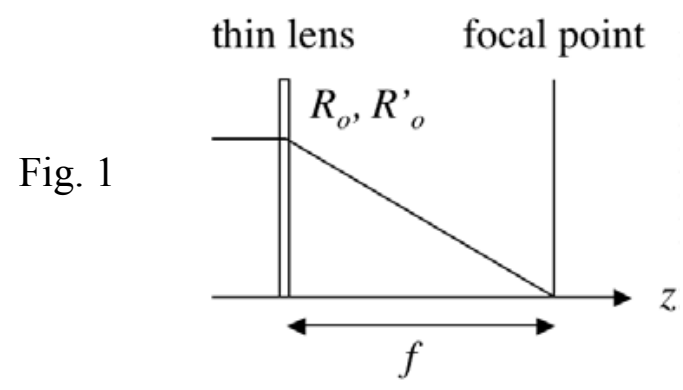

We can also determine the final spot size another way. Equation (I.2) can be rearranged as

$$
\frac{R^{\prime}}{\sqrt{R^{2}-E^{2} / C^{2}}}=-C,
$$

where $C^{2}=R_{o}^{\prime 2}+E^{2} / R_{o}^{2}$. Note that $R^{\prime}$ is negative for the convergent beam. Integrating Eq. (I.4) and then combing with Eq. (I.3) give us

$$
\sqrt{R^{2}-\frac{E^{2}}{C^{2}}}=\sqrt{R_{o}^{2}-\frac{E_{o}^{2}}{C_{o}^{2}}}-C z=R_{o}-C z .
$$

We have assumed $R_{o}\left|R_{o}{ }^{\prime}\right| \gg E$ again in the last step to get the final result in the above equation. We rewrite Eq. (I.5) to obtain the equation for the beam radius as

$$
R^{2} \approx \frac{E^{2}}{R_{o}^{\prime 2}}+\left(R_{o}-\left|R_{o}^{\prime}\right| z\right)^{2} .
$$

Let the distance between the lens and the focal point be $f$. Using the beam envelope parameters at the focal point $(z=f)$, i.e., $R=R_{f}$ and $R_{f}{ }^{\prime}=0$, Eq. (I.6) becomes

$$
R_{f} \approx \frac{E}{R_{o}} f \quad \text { and } \quad f \approx \frac{R_{o}}{\left|R_{o}^{\prime}\right|} .
$$

Note that $f$ in the above equation may not be the focal length of the system. Although the figure above shows a parallel, cold beam entering the thin lens, the derivation so far only uses the beam condition at the exit of the lens. A converging beam entering the system can be focused on to the same location by a weak focusing field with its final spot size still given by Eq. (I.4). 


\section{Spot Size and Chromatic Aberration}

Before we demonstrate how chromatic aberration causes spot size increase, we will make further simplification by assuming that the beam entering the focusing solenoid lens is parallel, i.e., $R_{o}{ }^{\prime}=0$. The focal length of the lens is given by $f=1 / \int k_{\beta}^{2} d z$, where $k_{\beta}=e B / 2 \gamma \beta m c^{2}$ is the betatron wavenumber. For a given focusing field, a small energy variation $(\Delta \gamma / \gamma)$ would lead to a focal length variation $(\Delta f / f)$ given by

$$
\frac{\Delta f}{f} \approx 2 \frac{\Delta \gamma}{\gamma},
$$

and the final spot size variation $\left(\Delta R / R_{f}\right)$ at the focal point $f$ given by

$$
\frac{\Delta R_{f}}{R_{f}} \approx \frac{\Delta f}{f} .
$$

Let us set the nominal focal length to be $f_{o}$. According to Eqs. (I.6), and (I.7), the spot size at the nominal focal point $\left(z=f_{o}\right)$ is given by Typically, a final focusing system would tightly focus the beam so that

$$
\begin{gathered}
R^{2} \approx R_{f}^{2}+\left.R_{o}^{2}\left(1-\frac{z}{f}\right)^{2}\right|_{z=f_{o}} \\
\approx R_{f o}^{2}\left(1+\frac{\Delta R_{f}}{R_{f o}}\right)^{2}+R_{o}^{2}\left[1-\left(1+\frac{\Delta f}{f_{o}}\right)^{-1}\right]^{2}, \\
\frac{\Delta \gamma}{\gamma} \gg\left(\frac{R_{f}}{R_{o}}\right)^{2} .
\end{gathered}
$$

For example, the above inequality is true for a beam in a radiography facility with a $\pm 1 \%$ energy variation, a $3-\mathrm{cm}$ beam radius entering the final focus lens and a 1-mm final spot size. With the condition given by Eq. (II.4), Eq. (II.3) can be written as

$$
R^{2} \approx\left(\frac{E f}{R_{o}}\right)^{2}+\left(2 R_{o} \frac{\Delta \gamma}{\gamma}\right)^{2}
$$

As shown in Fig. 2, the above equation indicates that there is a minimum spot size for a given focusing strength. To design a final focusing system, we can simply assume that the system will be operated near the optimal minimum spot size. Equation (II.5) can then be used to determine the input beam size, pipe size and the focal length of the system. 


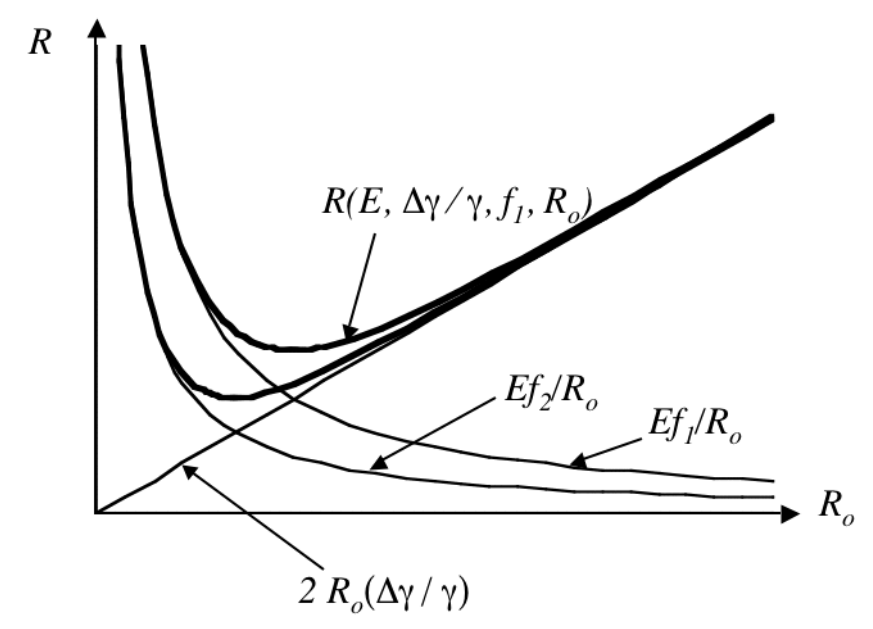

Fig. 2 The final spot size as a function of beam emittance, energy variation, entering beam radius and the focal length

\section{Spot Size and Spherical Aberration}

The magnetic field of a DC-like solenoid seen by a beam obeys the basic differential laws of magnetostatics, i.e.,

$$
\nabla \times \vec{B}=0
$$

and

$$
\nabla \bullet \vec{B}=0
$$

Since the azimuthally symmetric magnetic field only has components along the $\mathrm{z}$ axis and in the radial direction, Eq. (III.1) becomes

$$
\frac{\partial B_{r}}{\partial z}=\frac{\partial B_{z}}{\partial r}
$$

and Eq. (III.2) becomes

$$
\frac{1}{r} \frac{\partial}{\partial r}\left(r B_{r}\right)=-\frac{\partial B_{z}}{\partial z}
$$

By multiplying Eq. (III.4) by $r$ and then integrating it over $r$, we obtain

$$
B_{r}(r . z)=-\frac{1}{r} \int_{0}^{r} r \frac{\partial B_{z}}{\partial z} d r
$$

The solenoid's magnetic field on the axis only has one component which is $B_{z}(z, 0)$. Assume that the beam size is much smaller than the magnetic field's scale length. The magnetic field seen by the beam can then be expressed as 


$$
B_{z}(z, r) \cong B_{z}(z, o)-\frac{r^{2}}{4} \frac{\partial^{2} B_{z}(z, 0)}{\partial z^{2}}+\ldots
$$

and

$$
B_{r}(z, r) \cong-\frac{r}{2} \frac{\partial B_{z}(z, 0)}{\partial z}+\ldots
$$

The motion of an electron in an azimuthally symmetric magnetic field is given by

$$
\left\{\begin{array}{l}
\ddot{x}=-\frac{e}{\gamma m c}\left(\dot{y} B_{z}-\dot{z} B_{y}\right) \\
\ddot{y}=\frac{e}{\gamma m c}\left(\dot{x} B_{z}-\dot{z} B_{x}\right)
\end{array} .\right.
$$

Assuming that $d / d t=v_{z} d / d z$ and $d^{2} / d t^{2}=v_{z}^{2} d^{2} / d z^{2}$, Eq. (III.8) can now be written as

$$
\left\{\begin{array}{l}
x^{\prime \prime}=-\frac{e}{\gamma \beta_{/ /} m c^{2}}\left(y^{\prime} B_{z}-B_{y}\right) \\
y^{\prime \prime}=\frac{e}{\gamma \beta_{/ /} m c^{2}}\left(x^{\prime} B_{z}-B_{x}\right)
\end{array}\right.
$$

where $x^{\prime}=d x / d z$, etc. Let us set $\xi=x+i y$. Using the magnetic field given by Eqs. (III.6) and (III.7), we can express Eq. (III.9) in a single equation as

$$
\xi^{\prime \prime}=\frac{i e}{\gamma \beta_{\| /} m c^{2}}\left[\xi^{\prime}\left(B_{z o}-\frac{|\xi|^{2}}{4} B_{z o}^{\prime \prime}\right)+\frac{\xi}{2} B_{z o}^{\prime}\right],
$$

where $B_{z o}=B_{z}(z, 0)$. Let us rewrite the above equation in the Larmor frame by letting

$$
\xi=\Omega e^{i \int k_{\beta} d z}
$$

where $k_{\beta}$ is now given as

$$
k_{\beta}=\frac{e}{2 \gamma \beta_{l /} m c^{2}}\left(B_{z o}-\frac{|\Omega|^{2}}{4} B_{z o}^{\prime \prime}\right) .
$$

Note that $|\Omega|=|\xi|=r$. The equation of motion for an electron becomes

$$
\Omega^{\prime \prime}+k_{\beta}^{2} \Omega=0
$$

The nonlinear term hiding in $k_{\beta}$ is the source of the lens' spherical aberration. Instead of trying to solve Eq. (III.13) directly, we will use the thin lens approximation again. An 
electron ray with an initial radial displacement $\left(\Omega_{o}\right)$ and slope $\left(\Omega^{\prime}{ }_{o}\right)$ in the Larmor frame will leave the thin lens with the same radial displacement and a kicked slope given by

$$
\Omega^{\prime}=\Omega_{o}^{\prime}-\frac{\Omega_{o}}{f(|\Omega|)}
$$

where $f(|\Omega|)=f(r)$ is given by

$$
\begin{aligned}
f(r) & \cong\left[\int k_{\beta o}^{2}(z)\left(1-\frac{r^{2}}{2} \frac{B_{z o}^{\prime \prime}}{B_{z o}}\right) d z\right]^{-1} \\
& \cong f_{o}\left[1-\frac{r^{2}}{2} \frac{\int B_{z o} B_{z o}^{\prime \prime} d z}{\int B_{z o}^{2} d z}\right]^{-1},
\end{aligned}
$$

and $f_{o}$ is the focal length on the axis. Let us set

$$
\tilde{C}_{s}=\frac{\int B_{z o} B_{z o}^{\prime \prime} d z}{\int B_{z o}^{2} d z}
$$

Since the beam size is much smaller than the magnetic field's scale length, Eq. (III.12) can be expressed as

$$
f(r) \cong f_{o}\left(1+\frac{\tilde{C}_{s} r^{2}}{2}\right)
$$

At the focal point $z=f_{o}$, the ray's position is given by

$$
\Omega=\Omega_{o}-\Omega^{\prime} f_{o}
$$

Substituting Eqs. (III.11) and (III.14) into the above equation, we obtain

$$
\Omega=\Omega_{o}^{\prime} f_{o}+\frac{\Omega_{o} r_{o}^{2}}{2} \tilde{C}_{s}
$$

Squares of Eq. (III.16) gives the radial displacement of that individual electron ray. i.e.,

$$
r^{2} \cong r_{o}^{\prime 2} f_{o}^{2}+\frac{\tilde{C}_{s}^{2}}{4} r_{o}^{6}+\Omega_{o} \Omega_{o}^{\prime} r_{o}^{2} \tilde{C}_{s}
$$

The ensemble average of Eq. (III.17) over the entire beam gives the square of the RMS beam envelope. The ensemble average of the first term at the right is the square of the RMS beam divergence if the incoming beam envelope is parallel. For a uniformly 
distributed hard-edge beam, $\left\langle r_{o}{ }^{6}\right\rangle=2 R_{o}{ }^{6}$. The last term vanishes if the rays' positions and slopes are not correlated. Therefore, the RMS spot size at the focal point $f_{o}$ is given by

$$
R^{2} \cong\left(\frac{E}{R_{o}} f_{o}\right)^{2}+\left(C_{s} R_{o}^{3}\right)^{2}
$$

where the coefficient of spherical aberration $C_{s}$ is given by $C_{s}=\tilde{C}_{s} / \sqrt{2}$. Note that the constant factor in the coefficient changes with the beam distribution function. What is important to remember is the relationship between the spherical aberration coefficient and the magnetic field profile given by Eq. (III.13). We can include the contribution of chromatic aberration of the lens system to Eq. (III.18) by repeating the exercise presented in Sec. II. The scaling law for the final spot size due to the finite emittance, chromatic aberration and spherical aberration of the focusing system is then given by

$$
R^{2} \cong\left(\frac{E}{R_{o}} f_{o}\right)^{2}+\left(2 R_{o} \frac{\Delta \gamma}{\gamma}\right)^{2}+\left(C_{s} R_{o}^{3}\right)^{2}
$$

Figure 3 shows how the final spot size varying with the beam's initial beam size before it being focused by the final focusing lens. The contribution from each term in Eq. (III.20) is also plotted. Generally, the final focusing lens is designed to have a small spherical aberration. The spot size increase caused by the lens' spherical aberration is then insignificant for a nominal operation condition. For example, the ratio of the spherical aberration term to the emittance term in Eq. (II.20) is only about a few percent for a nominal DARHT-I beam and for a nominal FXR beam.

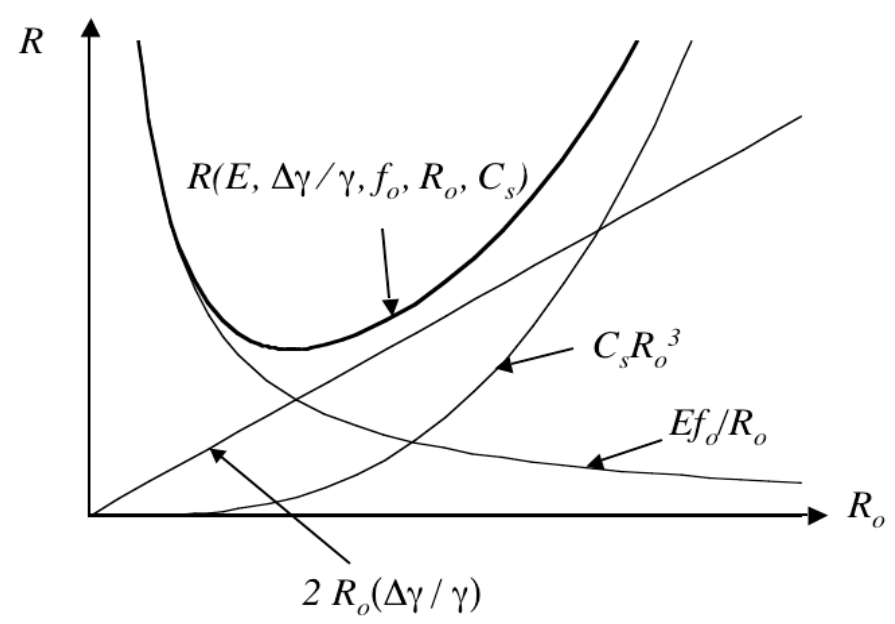

Fig. 3 The final spot size as a function of the initial beam radius. The contributions due to beam emittance, and chromatic aberration and spherical aberration of the focusing system are shown as well. 